\title{
Circulating Schistosoma DNA in seropositive patients: A two- step diagnostic approach to rule-out acute and active chronic schistosomiasis in low transmission settings
}

\section{Original Article}

\author{
Farouk G Gawish ${ }^{1}$, Ahmed MS Bayoumy ${ }^{2}$, Mohamed A Abd EI Raheem ${ }^{1}$, Anwar H Abo \\ Hashim $^{2}$, Ayman A El Badry ${ }^{3}$
}

Departments of Medical Parasitology, Faculty of Medicine, Al-Azhar Universities, Assiut ${ }^{1}$ and Cairo $^{2}$, Department of Clinical Microbiology, College of Medicine, Imam Abdulrahman Bin Faisal University, Dammam, Saudi Arabia ${ }^{3}$

\begin{abstract}
Background: Since its discovery in 1850 by Theodor Bilharz at Kasr Al-Ainy hospital, Cairo, Egypt, schistosomiasis continues to be a significant health challenge with a wide spectrum of chronic sequelae. There is no reliable single gold standard conventional method (microscopy or serology) for communities with low intensity and low transmission settings.

Objectives: To evaluate a two-step immunomolecular diagnostic approach to rule-out recent Schistosoma re-infection in patients with characteristic pathological radiological features of chronic schistosomiasis.

Subjects and Methods: Single serum samples were collected from patients from Kafr El Sheikh, Egypt. Selection of patients was based on microscopic absence of Schistosoma spp. eggs in either stool or urine samples; abstinence from treatment by Praziquantel in the last 2 months; and clinical and radiological diagnosis of pathological features of chronic schistosomiasis. All serum samples were serologically examined using indirect hemagglutination test (IHAT) for identification of anti-Schistosoma antibodies. Serum samples from 100 seropositive patients using IHAT were tested by multiplex PCR (mPCR) for detection of species-specific cell-free circulating Schistosoma DNA.

Results: None of the seropositive serum samples were positive by PCR targeting Schistosoma speciesspecific gene. Patients who were farmers and/or who had received Praziquantel treatment were associated with Schistosoma seropositivity with statistical significance.

Conclusion: A two-step immunomolecular diagnostic approach, using a single serum sample, can rule-out acute or active chronic schistosomiasis in patients that have characteristic pathological and radiological features of chronic schistosomiasis. Also, the two-step approach avoids over-treatment and development of drug resistance to Praziquantel, the only effective broad anti-Schistosoma medicine.
\end{abstract}

Keywords: circulating Schistosoma-DNA; Egypt; IHAT; PCR; praziquantel; schistosomiasis; seropositive.

Received: 1 March, 2021, Accepted: 17 March, 2021.

Corresponding Author: Ayman A. El Badry, Tel.: +966 133331168, E-mail: aelbadry@kasralainy.edu.eg

Print ISSN: 1687-7942, Online ISSN: 2090-2646, Vol. 14, No. 1, April, 2021.

\section{INTRODUCTION}

Schistosomiasis is a neglected tropical and subtropical helminthic parasitic disease caused by the Schistosoma spp. This neglected disease is multiconstituent including parasite, host, and vector factors. A successful massive drug administration (MDA) Schistosoma control program was implemented for the last 40 years in many countries including Egypt. However, due to re-infection schistosomiasis continues to be a significant health challenge, posing as one of the most disability-adjusted life years diseases and the cause of mortality in over 70 endemic countries in Africa, Middle East, South America and Asia $^{[1-4]}$.

Humans can be infected by two forms of Schistosoma infection, urogenital and intestinal, caused by five Schistosoma species: $S$. haematobium, $S$. mansoni, S. japonicum, S. intercalatum and S. mekongi. Schistosoma haematobium, mansoni, and japonicum are the main three species infecting humans worldwide. In Egypt, the two prevailing species are $S$. mansoni and haematobium including mixed infection by both species ${ }^{[3]}$. The resulting schistosomiasis is an acute parasitic infection that tends to persist as a life-long chronic debilitating disease. There is a wide spectrum of chronic sequelae of schistosomiasis, some of which are fatal, including chronic cystitis, carcinoma of the bladder, portal hypertension, pulmonary hypertension, male and female genital schistosomiasis, and cerebral schistosomiasis ${ }^{[5,6]}$.

The majority of the currently used diagnostic methods are conventional, including microscopic identification of Schistosoma ova in stool, urine and/or tissue smear, or immunological detection of parasitespecific antigen/antibodies ${ }^{[1,7,8]}$.

The MDA control program for schistosomiasis is based on the application of a treatment strategy for all populations living in Schistosoma endemic regions, 
whether they are infected or not. In Egypt, since 1996, a widespread MDA schistosomiasis control program has been applied. This control program maintains low Schistosoma transmission and low intensity of infection, engendering the challenge of missed cases of acute schistosomiasis based on parasitological microscopy. In addition to misdiagnosis, microscopy is of limited value in diagnosis of schistosomiasis during chronic infections with a wide spectrum of chronic disease sequelae $e^{[3,9,10]}$.

Diagnosing chronic schistosomiasis, in many countries including Egypt, is mainly dependent on serological diagnostictests, mainly IHAT, in the presence of characteristic radiological image features and in the absence of detectable Schistosoma ova in urine, stool samples or tissue biopsy. Serological tests that depend upon antibody detection cannot differentiate between recent and old infection, persistent and re-infection, or co-occurrence of the two or more Schistosoma species. They are also limited by the variability in sensitivity and specificity of different serological test formats. In addition, antibodies persist for months or even years following clearance of infection ${ }^{[7,8]}$.

Cell-free circulating Schistosoma-DNA are fragments detached from Schistosoma cells of a live-parasite and released in blood, saliva, urine, and other body fluids. Molecular detection of cell-free circulating SchistosomaDNA is used to detect early acute schistosomiasis and active chronic schistosomiasis in absence of Schistosoma eggs in stool and urine. These DNA fragments rapidly disappear within weeks following treatment making molecular methods a useful follow up tool ${ }^{[11-13]}$.

This study's intention was to evaluate a twostep immunomolecular diagnostic approach to rule-out Schistosoma recent re-infection in patients who had radiological characteristic pathological lesions of chronic schistosomiasis with a history of schistosomiasis or anti-Schistosoma treatment.

\section{SUBJECTS AND METHODS}

This laboratory-based cross-sectional study was carried out on serum samples from patients residing in Kafr El Sheikh from May, 2016 to February, 2017. Immuno- and PCR assays were carried out in the Diagnostic and Research Unit of Parasitic Diseases (DRUP), the Molecular Medical Parasitology Lab (LMMP), respectively, Department of Medical Parasitology, Faculty of Medicine, Cairo University, Egypt.

Patients: They were clinically and radiologically diagnosed with pathological features of chronic schistosomiasis and referred to the lab for serological testing for schistosomiasis. The exclusion criteria included patients less than 6 years old, patients with other underlying illnesses, patients for whom Schistosoma eggs were microscopically detected in their stool or urine samples and those who had taken Praziquantel in the last 2 months. A single blood sample was obtained from each patient, separated by centrifugation, then kept at $-20^{\circ} \mathrm{C}$ for immunoassays and DNA extraction, followed by PCR assay. Related patients' data were obtained with each sample and analyzed as predictors for Schistosoma seropositivity and molecular prevalence.

Immunoassay: Part of the serum from all study individuals was examined for detection of antiSchistosoma antibodies using IHAT (Fumouze Diagnostics, France) following the manufacturer's instructions.

Molecular assay[14,15]: Serum samples from 100 seropositive patients using IHAT were tested by mPCR assay. DNA extraction of all serum samples was done using QIAamp DNA Blood Mini Kit (Qiagen- Germany) following instructions by the manufacturer. The mPCR was used to amplify the extracted genomicDNA targeting Schistosoma-species specific DNA mitochondrial cytochrome oxidase I (mtDNA) gene. It can differentiate between $S$. haematobium and $S$. mansoni based on length of mPCR products. The MPCR was performed following the reaction condition, reaction mix and a set of 3 primers ${ }^{[14,15]}$, including common forward for Schistosoma (5 -TTT TTT GGT CAT CCT GAG GTG TAT-3), and 2 reverse primers for $S$. haematobium (5`-TGA TAA TCA ATG ACC CTG CAA TAA$3^{\prime}$ ) and $S$. mansoni ( $5^{\prime}$-TGC AGA TAA AGC CAC CCC TGT G-3'). The mPCR products (543 bp for $S$. haematobium and $375 \mathrm{bp}$ for $S$. mansoni) were electrophoresed on agarose gel $(1.5 \%)$ after staining the gel by ethidium bromide and viewed by a UV transilluminator.

Statistical analysis: Collected data was statistically analyzed using IBM SPSS V23 software. Patients associated quantitative variables were presented as mean and standard deviation; and the associated qualitative variables were tabulated as numbers and percentages. Variables were compared using the Chisquare test and/or Fisher exact test when appropriate. $P$ value equal to or less than 0.5 was considered significant.

Ethical Consideration: The study was started after approval by the ethical committee of the Faculty of Medicine, Al-Azhar University. Patients or their guardians were orally informed about the study goals; serum samples collection was done after acquiring their consent. Joining the study was voluntary.

\section{RESULTS}

A total of 100 patient serum samples were seropositive for schistosomiasis by IHAT. All seropositive 
patients met the inclusion and exclusion criteria of this study. Urine and stool specimens from included patients were microscopically negative for Schistosoma eggs. All sero-positive serum samples were PCR negative using PCR targeting Schistosomaspecies specific DNA.

The patient's characteristics were presented in table (1). Among seropositive patients, 75 (75\%) were males and 25 (25\%) were females. Those $>40$ -
50 years old were the most affected age group (42\%). The majority of seropositive patients were farmers (65\%), lived in rural areas (81\%) and had received Praziquantel treatment (68\%).

In our study, being a farmer $(P=0.01)$ and/or having received Praziquantel treatment $(P=0.02)$ were associated with Schistosoma seropositivity, with statistical significance (Table 1).

Table 1. Distribution of patients' characteristics in 100 seropositive Schistosoma patients.

\begin{tabular}{|c|c|c|c|c|}
\hline \multicolumn{2}{|l|}{ Patients' characteristics } & \multicolumn{2}{|c|}{ Seropositive (No. 100) } & \multirow{2}{*}{$\begin{array}{c}\text { Pvalue } \\
0.2\end{array}$} \\
\hline Age (Year) (Mean \pm SD) & & & & \\
\hline Age groups & $\begin{array}{l}20-30 \\
>30-40 \\
>40-50 \\
>50\end{array}$ & $\begin{array}{l}21 \\
25 \\
42 \\
12\end{array}$ & $\begin{array}{l}21.0 \% \\
25.0 \% \\
42.0 \% \\
12.0 \%\end{array}$ & 0.6 \\
\hline Sex & $\begin{array}{l}\text { Males } \\
\text { Females }\end{array}$ & $\begin{array}{l}75 \\
25 \\
\end{array}$ & $\begin{array}{l}75.0 \% \\
25.0 \% \\
\end{array}$ & 0.07 \\
\hline Occupation & $\begin{array}{l}\text { Farmer } \\
\text { Housewife } \\
\text { Worker } \\
\text { Unemployed } \\
\end{array}$ & $\begin{array}{c}65 \\
21 \\
11 \\
3\end{array}$ & $\begin{array}{c}65.0 \% \\
21.0 \% \\
11.0 \% \\
3.0 \%\end{array}$ & $0.01^{*}$ \\
\hline Residence & $\begin{array}{l}\text { Rural } \\
\text { Urban }\end{array}$ & $\begin{array}{l}81 \\
19 \\
\end{array}$ & $\begin{array}{l}81.0 \% \\
19.0 \% \\
\end{array}$ & 0.12 \\
\hline Praziquantel treatment & $\begin{array}{l}\text { Yes } \\
\text { No } \\
\end{array}$ & $\begin{array}{l}68 \\
32 \\
\end{array}$ & $\begin{array}{l}68.0 \% \\
32.0 \% \\
\end{array}$ & $0.02^{*}$ \\
\hline \multicolumn{2}{|c|}{ Total } & 10 & $100.0 \%$ & \\
\hline
\end{tabular}

\section{DISCUSSION}

Since its discovery in 1850 by Theodor Bilharz at Kasr Al-Ainy hospital, Cairo, chronic schistosomiasis continues as a significant health challenge worldwide, including Egyp $\mathrm{t}^{[3]}$. In the current study, circulating Schistosoma-DNA was not detected using PCR in all sera of seropositive patients with characteristic radiological imaging features of chronic schistosomiasis, supporting the idea that seropositivity of Schistosoma antibody among the studied population with chronic infection may be related to old Schistosoma infection. Serology alone is not a reliable diagnostic method for these patients, because it cannot rule out the possibility of reinfection or active chronic Schistosoma infection $^{[1,8,16]}$.

Characteristic radiological imaging features are important for diagnosing chronic schistosomiasis in the affected target organs, as well as assessing the complications and the severity of the disease. The primary target organs in schistosomiasis include urinary bladder in schistosomiasis haematobium, rectum and distal colon in schistosomiasis mansoni and schistosomiasis japonicum. The secondary target organs include the urinary tract, particularly the upper part, liver, spleen and lungs. In addition, accidentally Schistosoma eggs may deposit in ectopic secondary target organs and affect them via their venous anastomoses with the inferior vena cava. This results in genital schistosomiasis, cerebral schistosomiasis or skin ectopic infection. In chronic schistosomiasis the diagnosis is essentially by radiological imaging and confirmed by serology in absence of etiological microscopic diagnosis ${ }^{[16,17]}$. Serological tests, including IHAT, have many limitations: they cannot differentiate between recent/active Schistosoma and chronic old infections, persistent and re-infection, or co-infection of more than one Schistosoma species. They are also limited by variance in sensitivity and specificity of different serological test formats. In addition, antibodies persist for months to years after clearance of infection, which renders serological tests targeting antibodies useless in these circumstances ${ }^{[7]}$.

Rapid diagnostic test as a point of care test (POCT) is an easy applicable method that can detect specific circulating and secreted worm antigens. This test is easy, equipment-free, applicable, and rapid ${ }^{[18]}$. Schistosoma antigen-detection tests are limited by their false negative results due to low sensitivity and are not suitable for low infection intensity setting. However, they are not appropriate for epidemiological studies and monitoring MDA Schistosoma control programs ${ }^{[1,7,18]}$. This is especially true in underdiagnosed infections using low sensitive microscopy and antigen detection 
tests, or over diagnosis when using serological tests. Implementation of molecular diagnosis of schistosomiasis should not be limited to reference labs and should be readily available in small local and rural hospitals as well ${ }^{[7]}$.

Based on our results, in agreement with the results of different studies ${ }^{[1,7,8,16]}$, serological diagnosis is a poor positive test to rule-in the possibility of reinfection or active chronic schistosomiasis, and for those who were prescribed anti-Schistosoma treatment for characteristic radiological features of chronic schistosomiasis in target organs. Thus, we recommend a two-step approach for diagnosis of chronic schistosomiasis with late manifestations. Patients with a positive serological test should be further examined molecularly for Schistosoma-DNA, in order to avoid unnecessary treatment and the development of drug resistance to Praziquantel. Praziquantel is, to date, the only safe and effective anti-Schistosoma medicine, used for all types of human schistosomiasis as well as for control programs ${ }^{[4,10]}$.

This accurate, cost effective two-step active schistosomiasis diagnostic approach requires the implementation of molecular diagnosis in endemic areas, especially those in which DNA-based techniques, particularly PCR, is already available. Our study found a statistical significance association between Schistosoma seropositivity and being a farmer and/ or having received Praziquantel. No association was found between Schistosoma seropositivity using IAHT and the clinical picture, age group, sex and living in rural/urban setting. In endemic countries, both female and male gender predominance or non-predominance with Schistosoma infection was reported ${ }^{[19-22]}$. Children aged 5-17 years-old have been identified as the highest Schistosoma infected group in developing countries ${ }^{[3,22,23]}$. Additionally, farmers in contact with contaminated water when irrigating fields are in the highest risk occupation group for acquiring Schistosoma infection $^{[3,4,24]}$.

In conclusion, recent Schistosoma re-infection needs to be excluded in patients who have characteristic pathological and radiological features of chronic schistosomiasis and are living in low endemic regions with low transmission settings. For these patients, a two-step immunomolecular diagnostic approach (detection of cell-free circulating Schistosoma-DNA in sero-positive patients using a single serum sample) can rule-out acute or active chronic schistosomiasis. Also, it avoids unnecessary treatment and development of drug resistance to the only available effective broad anti-Schistosoma medicine, Praziquantel.

Author contribution: All authors made substantial contributions to the study design, analyzing, and interpreting data, revising the manuscriptand approved the final version submitted. Gawish FG, Bayoumy AMS,
Abd El Raheem MA, and Abo Hashim AH contributed to the collection of samples and to parasitological and immunological processing of samples. El-Badry AA conducted the molecular work for the study. Gawish FG drafted the manuscript and El-Badry AA wrote the final manuscript.

Acknowledgement: We would like to acknowledge chemist/Eman Abdul Gawad, Molecular Medical Parasitology Lab (LMMP), and chemist/Asmaa Ibrahim, Diagnostic and Research Unit of Parasitic Diseases (DRUP), Department of Medical Parasitology, Faculty of Medicine, Cairo University, Egypt, for contribution to the molecular work.

Conflict of interest: The authors declare no conflict of interest.

Funding statement: Not applicable, this research did not receive any funding.

\section{REFERENCES}

1. Colley DG, Bustinduy AL, Secor WE, King CH. Human schistosomiasis. Lancet 2014; 383:2253-2264.

2. Pisarski K. The global burden of disease of zoonotic parasitic diseases: top 5 contenders for priority consideration. Trop Med Infect Dis 2019; 4(1):44.

3. World Health organization (WHO) schistosomiasis fact sheet (2020). Available at https://www.who.int/newsroom/fact-sheets/detail/schistosomiasis. Accessed 6 March 2021.

4. Zacharia A, Mushi V, Makene T. A systematic review and meta-analysis on the rate of human schistosomiasis reinfection. PLoS One 2020; 15(12):e0243224.

5. Akhtar MM, ALJuhani N Sr, Younus D, ALSahafi AH, IV, Abouhamda A. Schistosomiasis mansoni manifesting as multiple colonic polyps. Cureus 2020; 12(11):e11755.

6. Cozzi D, Bertelli E, Savi E, Verna S, Zammarchi L, Tilli M, et al. Ultrasound findings in urogenital schistosomiasis: a pictorial essay. J Ultrasound 2020; 23(2):195-205.

7. Rabello AL, Garcia MM, Pinto da Silva RA, Rocha RS, Katz N. Humoral immune responses in patients with acute Schistosoma mansoni infection who were followed up for two years after treatment. Clin Infect Dis 1997; 24(3): 304-308.

8. Doenhoff MJ, Chiodini PL, Hamilton JV. Specific and sensitive diagnosis of schistosome infection: Can it be done with antibodies? Trends Parasitol 2004; 20(1):3539.

9. Elmorshedy H, Bergquist R, El-Ela NEA, Eassa SM, Elsakka EE, Barakat R. Can human schistosomiasis mansoni control be sustained in high-risk transmission foci in Egypt? Parasit Vectors 2015; 8(1):372

10. Ghazy RM, Tahoun MM, Abdo SM, El-Badry AA, Hamdy NA. Evaluation of praziquantel effectiveness after decades of prolonged use in an endemic area in Egypt. Acta Parasitol 2020. DOI: $10.1007 / \mathrm{s} 11686-020-00242$-x.

11. Lodh N, Naples JM, Bosompem KM, Quartey J, Shiff CJ. Detection of parasite-specific DNA in urine sediment 
obtained by filtration differentiates between single and mixed infections of Schistosoma mansoni and $S$. haematobium from endemic areas in Ghana. PLoS One 2014; 9(3):e91144.

12. Kato-Hayashi N, Leonardo LR, Arevalo NL, Tagum MNB, Apin J, Agsolid LM, et al. Detection of active schistosome infection by cell-free circulating DNA of Schistosoma japonicum in highly endemic areas in Sorsogon Province, the Philippines. Acta Tropica 2015; 141:178-183.

13. Weerakoon KG, Gordon CA, McManus DP. DNA Diagnostics for schistosomiasis control. Trop Med Infect Dis 2018; 3(3):81

14. Webster BL, Rollinson D, Stothard JR, Huyse T. Rapid diagnostic multiplex PCR (RD-PCR) to discriminate Schistosoma haematobium and S. bovis. J Helminthol 2010; 84(1):107-114.

15. Van den Broeck F, Geldof S, Polman K, Volckaert FAM, Huyse T. Optimal sample storage and extraction protocols for reliable multilocus genotyping of the human parasite Schistosoma mansoni. Infect Genet Evol 2011; 11(6):14131418.

16. Sah VK, Wang L, Min X, Rizal R, Feng Z, Ke Z, et al. Human schistosomiasis: A diagnostic imaging focused review of a neglected disease. Radiol Infect Dis 2015; 2(3):150-157.

17. Hatz CF. The use of ultrasound in schistosomiasis. Adv Parasitol 2001; 48:225-284.

18. Shiff C. Accurate diagnostics for schistosomiasis: a new role for PCR? Rep Parasitol 2015; 4:23-29.

19. Remoué F, To Van D, Schacht AM, Picquet M, Garraud O, Vercruysse J, et al. Gender-dependent specific immune response during chronic human schistosomiasis haematobia. Clin Exp Immunol 2001; 124(1):62-68.

20. Satayathum SA, Muchiri EM, Ouma JH, Whalen CC, King CH. Factors affecting infection or reinfection with Schistosoma haematobium in coastal Kenya: survival analysis during a nine-year, school-based treatment program. Am J Trop Med Hyg 2006; 75(1):83-92.

21. Rudge JW, Stothard JR, Basáñez MG, Mgeni AF, Khamis IS, Khamis AN, et al. Micro-epidemiology of urinary schistosomiasis in Zanzibar: Local risk factors associated with distribution of infections among schoolchildren and relevance for control. Acta Trop 2008; 105(1):45-54.

22. Kalinda C, Mutengo M, Chimbari M. A meta-analysis of changes in schistosomiasis prevalence in Zambia: implications on the 2020 elimination target. Parasitol Res 2020; 119(1):1-10.

23. Lim RM, Woolhouse MEJ, Mduluza T, Chase-Topping $\mathrm{M}$, Osakunor DNM, Chitsulo L, et al. Investigating a strategy for quantifying schistosome infection levels in preschool-aged children using prevalence data from school-aged children. PLoS Negl Trop Dis 2020; 14(10): e0008650.

24. Nyati-Jokomo Z, Chimbari MJ. Risk factors for schistosomiasis transmission among school children in Gwanda district, Zimbabwe. Acta Trop 2017; 175:8490. 